\title{
Comparison of three different heuristics for laser heat treatment pattern design
}

\author{
Axel Nordin ${ }^{1}$ \\ ${ }^{1}$ Lund University \\ axel.nordin@design.lth.se
}

\begin{abstract}
Keywords:

\section{Introduction}

To reduce weight and emissions, the automotive industry has developed a large number of innovative processes for achieving light but strong components. One such innovation is hot stamping, first developed and patented by Plannja in 1977 (GB1490535, 1977) and later used with boron steel in the automotive industry by Saab Automotive AB in 1984 (Berglund, 2008). However, hot stamping has some drawbacks, compared to regular stamping, such as increased difficulty in post-trimming operations, higher energy consumption, expensive specialized forming tools with cooling and the need of having a heating step in-line with the rest of the production line. These shortcomings could potentially be eliminated by instead selectively heat treating the part by means of a heating source, such as a laser or an induction heating element. In this study, local heat treatment of boron steel, as described in (Asnafi et al., 2016; Sagar et al., 2018), is investigated in terms of its effect on final part structural performance. In order to optimize the properties of the heat-treated part, the location of the heat treatment pattern must be optimized. The objective is to evaluate different methods for achieving an optimal heat treatment pattern. The performance metric in all cases is how well the selectively heat treated part performs compared to a fully heat treated part in terms of structural performance and the area heat treated (in order to minimize cycle time and energy consumption).

Three different methods for determining the optimal heat treatment pattern have been developed and are described in this paper. Each method is evaluated using an initial benchmark geometry and load case (see Section 3.1). Based on the results from the benchmark, one of the pattern generation methods is selected for further validation using a complex component and load case (see Section 4.1).

This paper is structured as follows. In Section 2, the process and material used in this study are described. In Section 3, the pattern generation heuristics are described and evaluated using a benchmark. In Section 4, one of the heuristics is applied to a more complex component and load case. 


\section{Background}

\subsection{Related works}

Local heat treatment of various metals for improved formability has been investigated previously by several authors, (see e.g. Merklein, Johannes, Lechner, \& Kuppert, 2014). However, few have considered the effect of local heat treatment on the performance of the final part. Conrads et al. (Conrads, Daamen, Hirt, \& Bambach, 2016) investigated the effects of local heat treatment of high manganese steel on the crashworthiness of a test geometry. Asnafi et al. investigated the formability of parts in boron steel heat treated with a laser source (Asnafi, Andersson, Persson, \& Liljengren, 2016) and Sagar et al. investigated the geometric variation of parts produced using the same process (Sagar, Wärmefjord, \& Söderberg, 2018).

\subsection{Underlying principle}

Heat treatment of sheet materials aims to improve properties such as the formability and/or strength of the material. For the material considered in this study, boron sheet steel, heat treatment can be used to create areas with martensite. The hardened martensitic areas can add strength to the overall part, depending on where they are located. The pattern of hardened areas needs to be optimized, based on the intended use of the end product.

\subsection{Material}

The material used in this study is $1 \mathrm{~mm}$ Boloc 02 boron steel (Duroc Special Steel AB, 2009). The material composition can be found in Table 1. The material properties can be found in Table 2.

Table 1. Material composition of Boloc 02 (Duroc Special Steel AB, 2009)

\begin{tabular}{lllllll}
$\mathbf{C} \%$ & $\mathbf{S i} \%$ & Mn\% & P\% & S\% & Cr\% & B\% \\
\hline \hline $.2-.25$ & $.2-.35$ & $1-1.3$ & .003 & .01 & $.14-.26$ & $.0015-.005$
\end{tabular}

Table 2. Mechanical properties for Boloc 02 (Duroc Special Steel AB, 2009)

\begin{tabular}{lllll} 
Condition & Re MPa & Rm MPa & e A80 \% & HV \\
\hline \hline Annealed & 340 & 480 & 28 & 140 \\
Hardened & & 1590 & 6 & 520 \\
Water & & & &
\end{tabular}

\subsection{Material modelling}

The material model is based on the assumption that only the yield point of the material is affected by the heat treatment (no failure modelling). The material model is based on a simple bi-linear isotropic hardening model, where the tangential modulus and yield stress have been empirically fit to experimental data. The material properties used in the following simulations can be found in Table 3.

Table 3. Material model

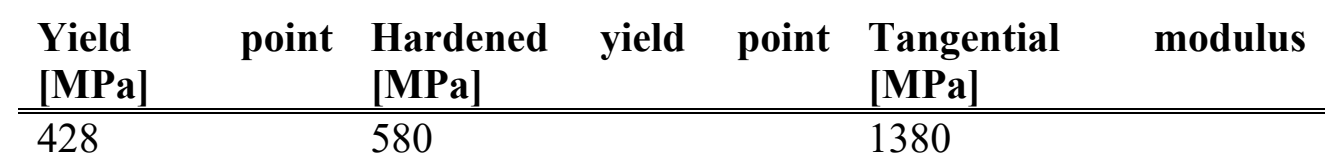




\subsection{Heat treatment modelling}

The application of the heat treatment pattern is achieved using a coupled thermo-mechanical simulation. A steady-state thermal simulation is used to determine the heat affected zones based on the input pattern. The thermal simulation is only used to find the heat affected areas and does not model the thermal history of the material. A material model with different yield strengths depending on the temperature is used in the mechanical simulation. The coefficient of thermal expansion is artificially set to 0 for the material to avoid unintended effects of thermal strains in the mechanical simulation.

\section{Evaluation of pattern generation heuristics}

Three pattern generation heuristics have been developed and are compared in this paper:

1) Topology optimization heuristic

2) Principal stress line heuristic

3) Plastic strain heuristic

The heuristics are compared based on a simple benchmark geometry and load case, as described in Section 3.1. The objective in both cases is to maximize the force needed to deform the part a set distance, as this is easily measured in physical validation tests, and is of relevance to the potential applications of this method. The optimal method has the highest reaction force and lowest heated area. However, since this is a multi-objective optimization problem with opposing goal functions, a prioritization of the objectives needs to be made. In this case, the reaction force increase compared to the nominal design divided by the area heated was selected as a combined goal function to reduce the problem, as shown in Eq. 1.

$$
\max \left(\frac{F_{\text {reaction,method }}-F_{\text {reaction,nominal }}}{A_{\text {heated,method }}}\right)
$$

\subsection{Initial Benchmark}

The methods are compared using a simple tensile test specimen. The specimen is shown in Figure 1 and Figure 2. In the load case, all degrees of freedom are locked at one end, and a displacement of $2 \mathrm{~mm}$ is applied to the other. The specimen is modelled in ANSYS Mechanical as a surface body meshed with shell elements with a thickness of $1 \mathrm{~mm}$, as shown in Figure 3 . The reaction force of the forced displacement is measured. The analysis is a non-linear static structural analysis taking into account large strains and the bi-linear plasticity material model described in Section 2.4. 


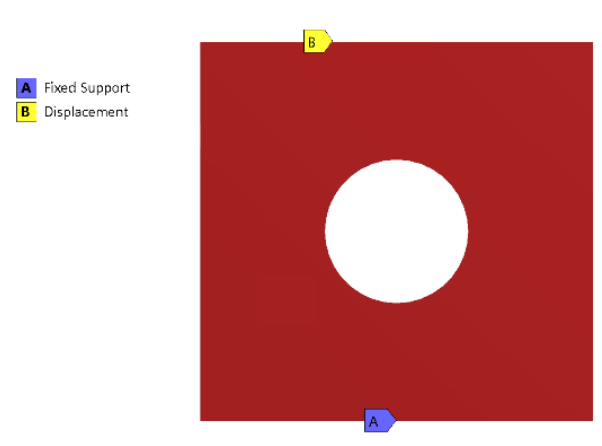

Figure 1. Benchmark geometry and load case

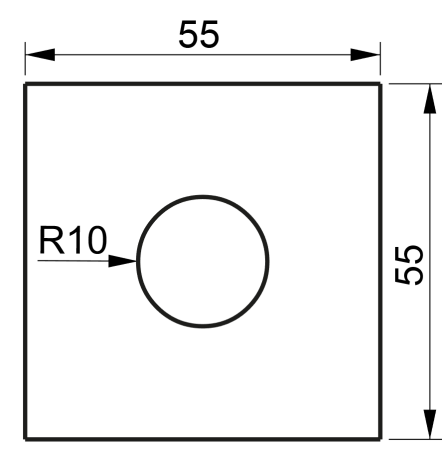

Figure 2. Dimensions of benchmark geometry

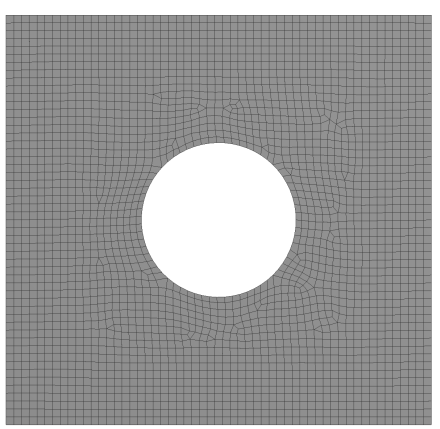

Figure 3. Test specimen mesh

\subsection{Topology optimization}

The first method is based on topology optimization, as first introduced by Bendsøe and Kikuchi (1988). The most common use of topology optimization is to distribute material within a design space to maximize or minimize certain objectives. A review of different topology optimization approaches can be found in (Sigmund \& Maute, 2013).

For the purpose of optimizing the heat treatment pattern, where the material is not redistributed but rather only modified, a heuristic based on topology optimization is developed. The assumption is that a topology optimization will generate a material distribution that will be a good estimation of where the heat treatment should be performed to increase its strength.

Each step of the heuristic is described in detail in the following sections. The topology optimization heuristic can be summarized as follows:

1. Perform a topology optimization based on the load case

2. Apply heat treatment where material exists in the topology optimization result

3. Perform steady-state thermal simulation to get temperature results

4. Import temperature distribution into structural analysis with temperature dependent material data

5. Perform structural analysis

\subsubsection{Perform a topology optimization}

The topology optimization is based on the same load case as the part will be subject to in the real analysis, see Section 3.1. The mesh size is chosen to so that the heating pattern can be resolved, which for the benchmark was determined to be $1 \mathrm{~mm}$.

The mass is constrained to a certain percentage of the original and the objective is set to minimize compliance (i.e. maximize stiffness). In the case of the benchmark, the mass percentage was set to $20 \%$, that is, a heat treatment area of $20 \%$.

To take into account that the heat treatment will only change the material properties and not the material distribution in the part, the minimum pseudo-density is modified to mimic this behavior. The pseudo-density is used as a scaling factor for Young's modulus in the material. A pseudo-density of 0 would represent a complete void. However, since no material will be removed in the heat treatment, a void would not be representative of the material. Instead, the minimum pseudo-density, $\rho_{\min }$, is based on the relation between the tangent modulus of the plasticized material, and Young's modulus, as described by Eq. 2. The reasoning is that the material that is heat treated will not plasticize, but rather remain in the linear region, whereas the non-heat-treated material will plasticize and its stiffness will be proportional to the tangent modulus of the bi-linear material model. As the tangent modulus, $E_{t}$, for the material in the benchmark part was estimated to be $1.380 \mathrm{GPa}$, and Young's modulus, $E$, to be $210 \mathrm{GPa}$, the ratio between the two is $1.38 / 210$, or $6.6 \mathrm{e}-3$. The minimum pseudo-density was therefore set to 
6.6e-3, and the maximum to 1 . Other simulation settings, such as the convergence limit and number of iterations were left in their default settings.

$$
\rho_{\min }=\frac{E_{t}}{E}
$$

\subsubsection{Apply thermal loads at locations where material exists in the topology optimization result}

The resulting material distribution from the converged topology optimization is exported as an STL-file, which contains a triangulated representation of the material distribution, and processed using a Matlab-script to output nodal temperature data for the next step of the simulation. The script takes the locations of the vertices in the STL-file and applies a thermal load to those nodes.

\subsubsection{Perform steady-state thermal simulation to get temperature results}

Using the External Data component in ANSYS Workbench, the thermal loads can be interpolated onto the mesh of the part. A higher mesh density may be employed to help interpolate the imported temperature load. In the study, a mesh size of $1 \mathrm{~mm}$ was used for the topology optimization, and a mesh size of $0.25 \mathrm{~mm}$ was used for the thermal simulation.

\subsubsection{Import temperature distribution into structural analysis with temperature dependent material data}

The results from the thermal simulation are interpolated onto the mesh for the structural simulation to establish the material properties of the heat treated sheet. The reaction force is then solved for as described in Section 3.1.

\subsection{Principal Stress Line Heuristic}

The second method is based on principal stress vectors. One reason behind using principal stress directions as the driving force behind the generated heating pattern is that these can be used to find the load paths under tensile and compressive stress in the material. This method has previously been used to design components in e.g. (Kokcharov \& Burov, 2001; Kwok, Li, \& Chen, 2016; Li \& Chen, 2010; Tam \& Mueller, 2015). Another reason behind using this heuristic is that the width of the generated pattern can easily be controlled to correspond to the width of, for instance, the laser spot width used for heating.

The Principal Stress Line Heuristic can be summarized as follows:

1. Perform a structural analysis of non-heat-treated part to obtain principal stress vectors

2. Calculate the principal stress lines from the stress vectors

3. Offset the principal stress lines to the thickness of the laser

4. Apply thermal loads at locations where principal stress lines exist

5. Perform steady-state thermal simulation to get temperature results

6. Import temperature distribution into structural analysis with temperature dependent material data

7. Perform structural analysis of heat-treated part

\subsubsection{Perform a structural analysis to obtain principal stress vectors}

A structural analysis is performed in ANSYS Mechanical, using the load case from Section 3.1. The resulting principal stress vectors are exported as nodal results and imported into the nodebased scripting component Grasshopper in McNeel Rhinoceros (Robert McNeel \& Associates, 2014, 2015). 


\subsubsection{Principal stress lines}

In Grasshopper, the structural optimization plug-in Millipede (Michalatos \& Kaijima, 2017) is used to generate the principal stress lines based on the imported stress vectors. The number of principal stress lines and the starting positions are user defined. The stress lines follow the direction of the principal stress vectors. The lines are then offset to correspond to the thickness of the heating source. The resulting heating pattern is exported as an STL file and read by the same script that interpreted the topology optimization results, as described in Section 3.2.2.

\subsubsection{Apply heating pattern and perform structural analysis}

The next parts of the process are identical to steps 3-5 in the topology optimization heuristic.

\subsection{Plastic Strain Heuristic}

The third method is based on only hardening the areas that experience plastic strain. As the heat treatment only affects the yield strength, there is no need to heat treat any other parts. However, since heat treating one area will affect how the rest of the material behaves under loading, the areas that experience plastic strains will also change. This necessitates an iterative approach to be taken.

The plastic strain approach is thus performed by first running a structural analysis with no heat treatment to find the initial strain distribution. The strain is then exported to a script which finds the most affected areas of the material and creates a thermal load input file based on the data. The most affected areas are determined by setting a percentage of the total area that should be heated, and then distributing that area where the highest strains are. This thermal load is then imported and used for a thermal analysis, just as in the previous methods. The heat treated part is analyzed in the structural simulation, and the strain data is once more exported. The strain data from iteration 1 and 2 is then read in by the script and used to construct the new temperature load. This process is continued until there is little change between iterations. To avoid removing areas that were heat treated in iteration $i-1$ in iteration $i$, a history of the heat treated areas is kept.

The pseudocode for the script that interprets the strain data is as follows:

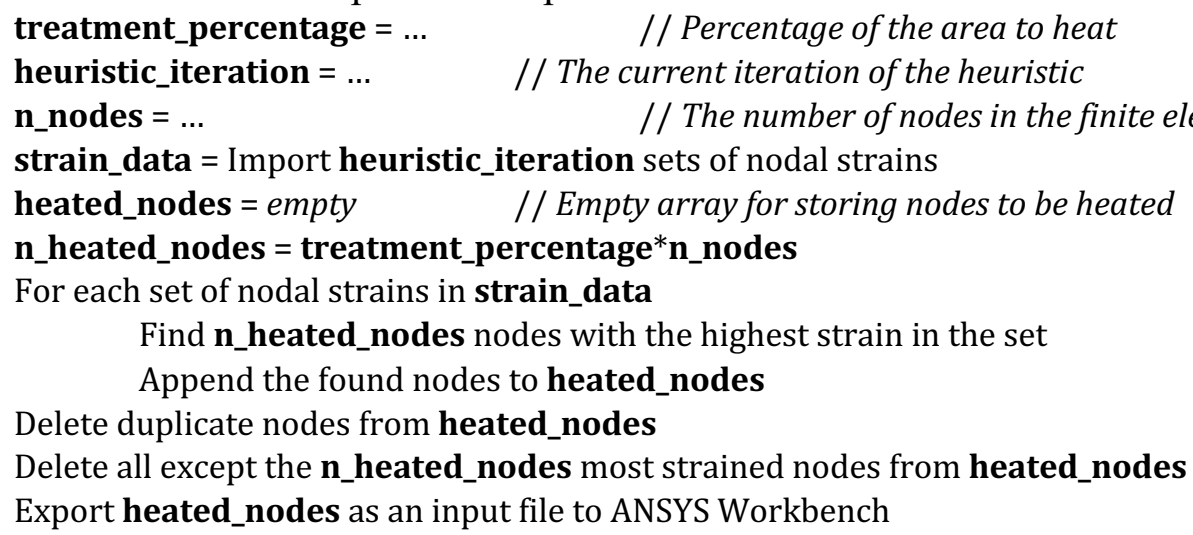

The plastic strain heuristic can be summarized as follows:

1. Perform a structural analysis of non-heat treated part to obtain strain

2. Apply thermal loads in the areas with the highest strain

3. Perform steady-state thermal simulation to get temperature results

4. Import temperature distribution into structural analysis with temperature dependent material data

5. Perform a structural analysis of heat-treated part to obtain strain

6. Repeat step 2-5 until the heat treatment pattern does not change between iterations 


\subsection{Results}

The three heuristics were applied to the simple tensile test shown in Figure 1. The reaction force was measured and the area heated by the method was calculated. The results were compared to a nominal, unhardened part, and a fully hardened part. The results are shown in Table 4 and Figure 4. The generated heating patterns for the three heuristics are shown in Figure 5. The principal stress heuristic gives the highest value for Eq. 1.

Table 4. Results for the three different heuristics for a tensile test specimen

\begin{tabular}{llll} 
Heuristic & Reaction force increase & Area heated & Objective function \\
\hline \hline Nominal & $0 \mathrm{~N}$ & $0 \%$ & N/A \\
Topology optimization & $802 \mathrm{~N}$ & $45 \%$ & 1782 \\
PSL & $2029 \mathrm{~N}$ & $74 \%$ & 2742 \\
PS (Iteration 1) & $1613 \mathrm{~N}$ & $19 \%$ & 8489 \\
PS (Iteration 2) & $1715 \mathrm{~N}$ & $19 \%$ & 9026 \\
Fully hardened & $2494 \mathrm{~N}$ & $100 \%$ & 2494
\end{tabular}

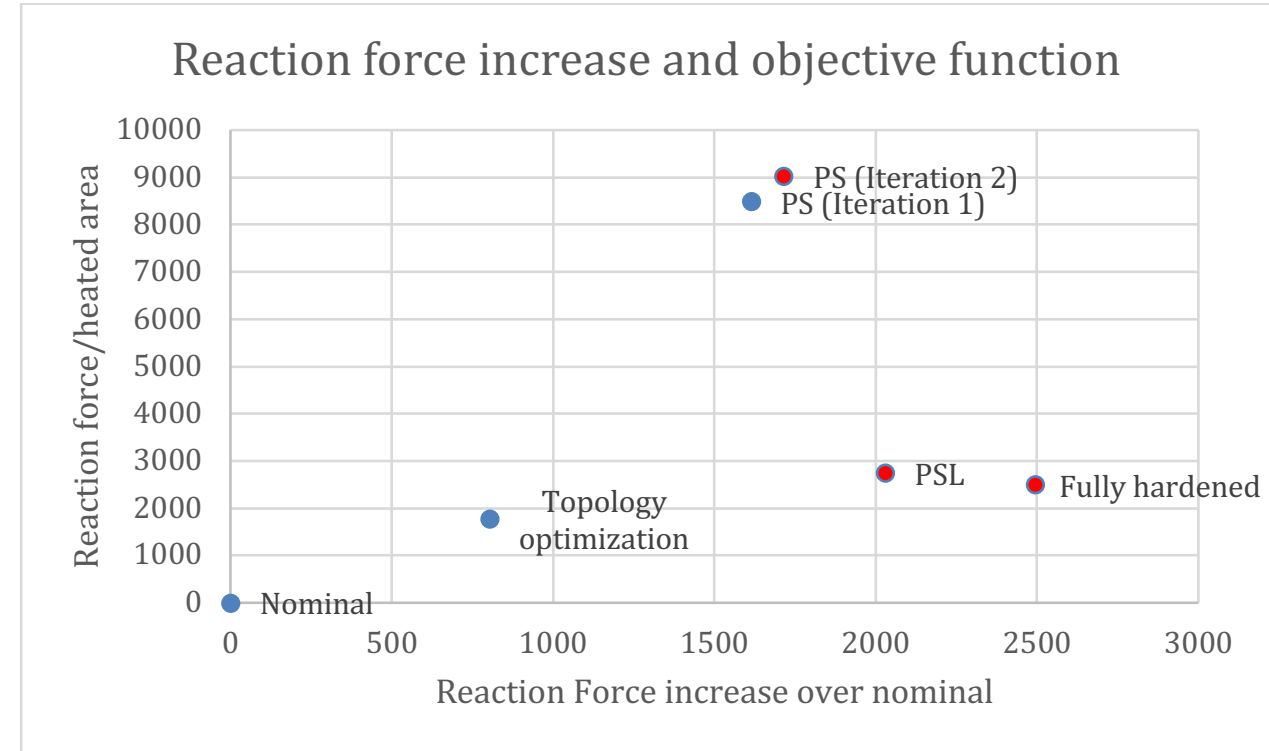

Figure 4. Comparison of the three methods

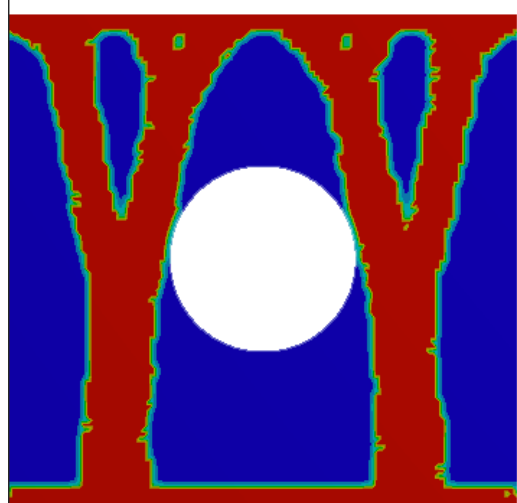

Principal stress line

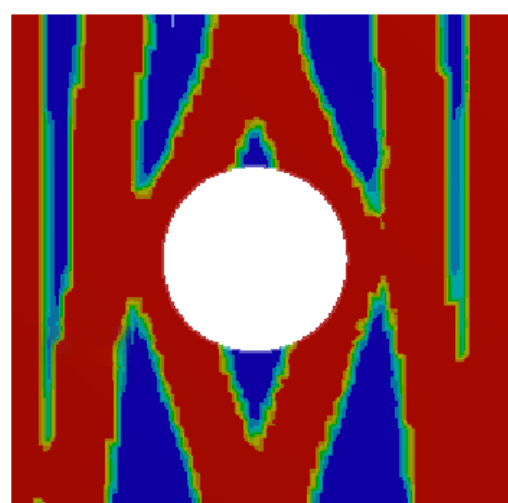

Topology optimization

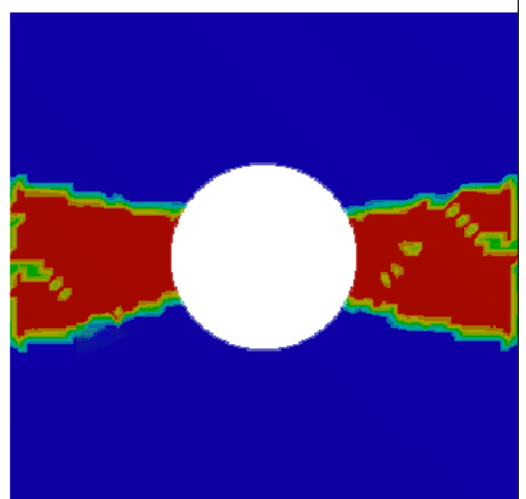

Plastic strain heuristic

Figure 5. Heating patterns for the three methods 


\section{Optimization of a complex component}

To validate the approach on a more complex geometry and load case, the plastic strain heuristic was applied to another component. The plastic strain heuristic was chosen as it gave the highest value for the goal function described by Eq. 1.

\subsection{Component}

The component is a beam based on a flex-rail geometry made from $1 \mathrm{~mm}$ Boloc 02 boron sheet steel, the same material used for the previous validation tests. The flex-rail geometry is described in (Andersson, 2007). The beam is formed in a test mold at RISE IVF in Olofström, Sweden, based on the results from previous studies into the formability of the heat treated material, found in (Asnafi et al., 2016; Sagar et al., 2018). The overall dimensions are shown in Figure 7. The objective for the optimization was to increase the pressing force required to deform the beam by $30 \mathrm{~mm}$.

\subsection{Physical test setup}

The beam was subjected to a three point bending test, with the pressing force and displacement being measured. The physical test setup is shown in Figure 8.

An initial physical test with a formed beam from Boloc 02 material was first conducted to measure the nominal press force as a function of press displacement. Based on the physical test data, a virtual model was set up and simulated using the same conditions to find the strains of the nominal design.

\subsection{Structural analysis of nominal part}

The simulation was run as a non-linear static structural analysis in ANSYS Workbench, taking into account large strains. The beam was modelled as a surface body using shell elements, welded to a surface body plate and supported by two solid cylinders. The setup is shown in Figure 6. A frictionless contact, allowing sliding tangentially and separation in the normal direction, was defined between the cylinders and the beam.

The pressing was modeled through a forced displacement of the top cylinder by $30 \mathrm{~mm}$. To constrain the model, the circular faces on the ends of the bottom cylinders were set as fixed in all degrees of freedom. The end surfaces of the top cylinder were constrained from moving in any other direction than downwards. To constrain the beam and restrict rigid body motion due to the frictionless contacts, two vertices were constrained from moving in the x-direction, and two other vertices were constrained from moving in the y-direction. The top and bottom cylinders restricted the z-translation. The cylinders were modeled as having a linear material model to reduce the computational complexity. 
A Fixed Support

B Displacement

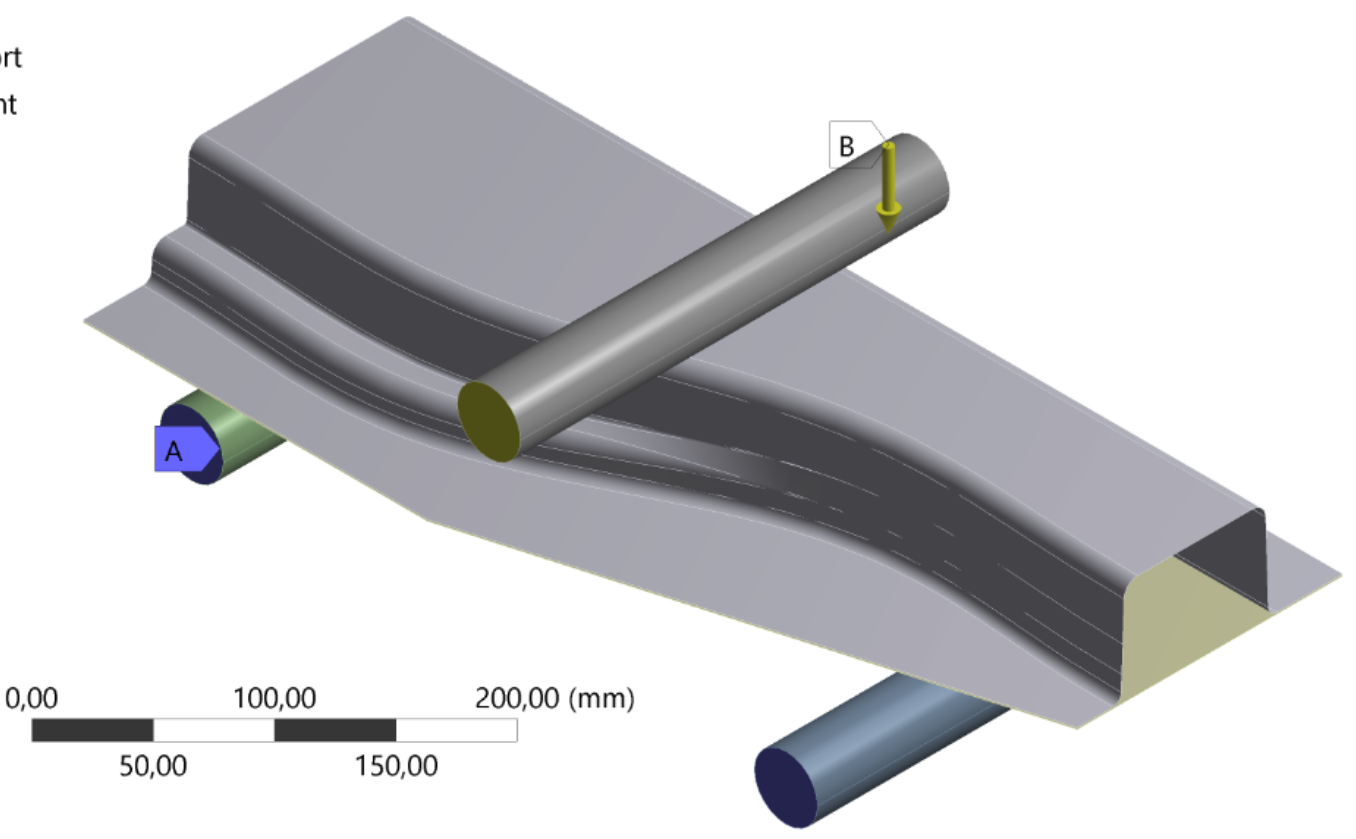

Figure 6. The applied boundary conditions

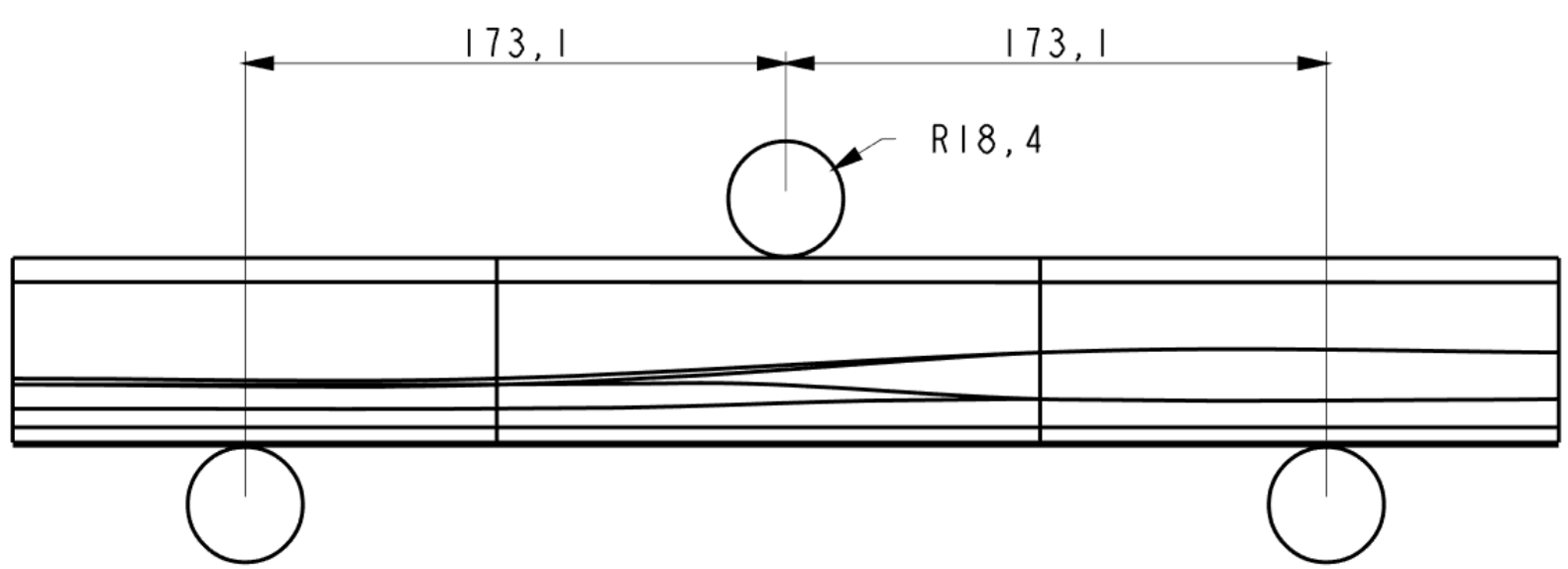

Figure 7. Dimensions of the test setup

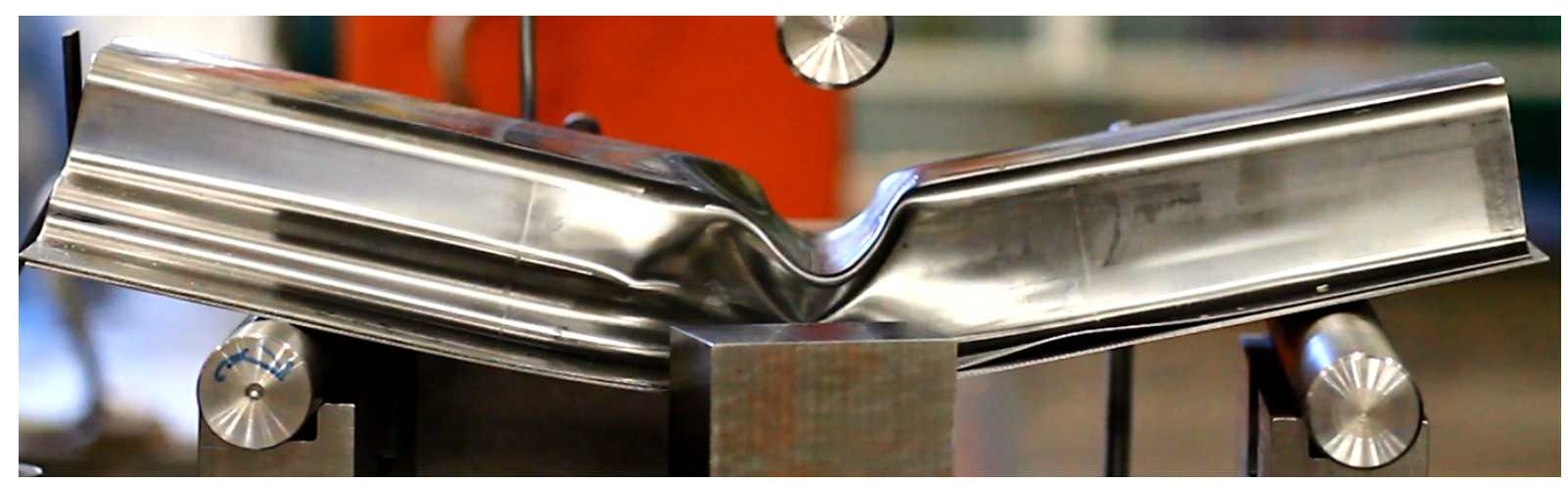

Figure 8. The physical test setup. Courtesy of RISE IVF

\subsection{Apply the Plastic Strain Heuristic}

The plastic strains from the simulation were then used to determine where the heat treatment should be performed, as described in Section 3.4. The areas with the highest strain were 
designated to be heat treated. The maximum heat-treatment area was constrained to $20 \%$ of the total area.

The resulting heating pattern was then applied, and the structural analysis was performed again.

\subsection{Results}

After two iterations, the change in the heating pattern was small and the heuristic was stopped. The resulting heating pattern is shown in Figure 9.

The simulation predicted a reaction force that was $85 \%$ of the fully hardened beam, as shown in Table 5.

Table 5. The reaction force and area heated for the different variants

\begin{tabular}{|c|c|c|c|c|}
\hline Heuristic & $\begin{array}{l}\text { Reaction } \\
\text { increase }\end{array}$ & force & Area heated & Objective function \\
\hline Nominal & $0 \mathrm{~N}$ & & $0 \%$ & N/A \\
\hline PS 1 & $1169 \mathrm{~N}$ & & $20 \%$ & 5845 \\
\hline PS 2 & $1286 \mathrm{~N}$ & & $22 \%$ & 5845 \\
\hline Fully hardened & $1507 \mathrm{~N}$ & & $100 \%$ & 1507 \\
\hline
\end{tabular}

Figure 9. a) Plastic strains for the unhardened material. b) Resulting heating pattern \#1. c) Plastic strains for heating pattern \#1. d) Resulting heating pattern \#2

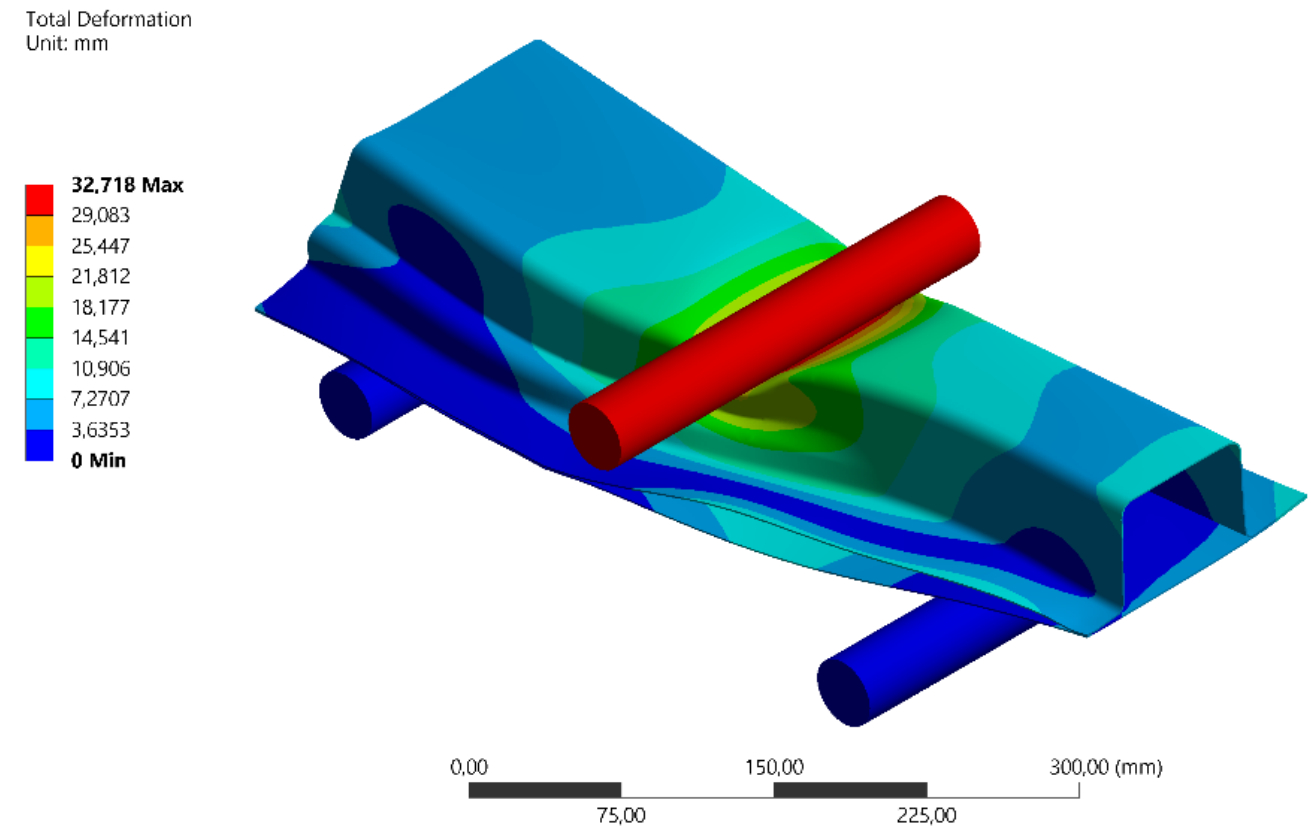

Figure 10. Image of the deformed state of the beam 


\section{Discussion}

The results from Section 3.5 indicate that the plastic strain heuristic performs best based on the objective function described by Eq. 1 and that the heuristic also heats the least area. However, the principal stress line heuristic results in a higher reaction force compared to the plastic strain heuristic and also outputs paths that can easily be followed by a numerically controlled tool, such as a laser, for heat treatment. Moreover, the width of the resulting heating pattern from the principal stress line heuristic can be controlled. A drawback to the principal stress line heuristic, apart from the lower objective value, is that the generated lines are sensitive to the initial conditions, such as the number of stress lines to be generated and any singularities in the principal stress vector result. Overall, the plastic strain heuristic is thus the most efficient, automated and robust heuristic of the three tested in this paper, especially if other local heating methods, such as induction, are used.

\section{Conclusion}

This paper describes three heuristics for generating heat treatment patterns for selective hardening of boron steel sheets. The results show that an algorithm based on a plastic strain heuristic, as described in this paper, achieves part performance close to that of hot stamping while only heating one-fifth of the surface in a demonstrator part from the automotive industry. While the results are promising, further physical validation is needed and the link between optimization results and application of the heat treatment pattern needs to be further investigated. Other heat treatment methods, such as induction heating, and other materials, such as aluminum, are currently being evaluated. This paper has only evaluated the effects of localized heat treatment on the reaction force of two components and future research should also investigate how other aspects, such as buckling and energy absorption may be affected by local heat treatment.

\section{Acknowledgements}

This study has been made possible through a grant from the AForsk foundation (16-549). The study has been carried out in collaboration with VA Automotive and RISE IVF in Olofström who conducted the mechanical testing and metal forming.

\section{References}

Andersson, A. (2007). Numerical and Experimental Evaluation of Springback in Advanced High Strength Steel. Journal of Materials Engineering and Performance, 16(3), 301307. https://doi.org/10.1007/s11665-007-9056-9

Asnafi, N., Andersson, R., Persson, M., \& Liljengren, M. (2016). Tailored boron steel sheet component properties by selective laser heat treatment. IOP Conf. Ser.: Mater. Sci. Eng., 159(1). https://doi.org/10.1088/1757-899X/159/1/012023

Bendsøe, M. P., \& Kikuchi, N. (1988). Generating optimal topologies in structural design using a homogenization method. Computer Methods in Applied Mechanics and Engineering, $71(2), 197-224$.

Berglund, G. (2008). The history of hardening of boron steel in northern Sweden. In 1st International Conference on Hot Sheet Metal Forming of High-Performance Steel (pp. 175-177).

Conrads, L., Daamen, M., Hirt, G., \& Bambach, M. (2016). Improving the crash behavior of structural components made of advanced high strength steel by local heat treatment. IOP Conf. Ser.: Mater. Sci. Eng, 159(012024). https://doi.org/10.1088/1757$899 \mathrm{X} / 159 / 1 / 012024$

Duroc Special Steel AB. (2009). Kallvalsade och glödgade borstål för vatten eller oljehärdning. 
Kokcharov, I. I., \& Burov, A. E. (2001). Analysis of stress state with the force lines method. In Proceedings of International Conference RDAMM 2001 (Vol. 6, pp. 208-212).

Kwok, T.-H., Li, Y., \& Chen, Y. (2016). A structural topology design method based on principal stress line. Computer-Aided Design, 80, 19-31.

Li, Y., \& Chen, Y. (2010). Beam Structure Optimization for Additive Manufacturing Based on Principal Stress Lines. In Proceedings of the 21st Annual International Solid Freeform Fabrication Symposium (pp. 666-678). SFF.

Merklein, M., Johannes, M., Lechner, M., \& Kuppert, A. (2014). Journal of Materials Processing Technology A review on tailored blanks - Production, applications and evaluation. Journal of Materials Processing Tech., 214(2), 151-164. https://doi.org/10.1016/j.jmatprotec.2013.08.015

Michalatos, P., \& Kaijima, S. (2017). Millipede. Retrieved from www.grasshopper3d.com/group/millipede

Norrbottens Jaernverk AB. (1977). GB1490535.

Robert McNeel \& Associates. (2014). Grasshopper [Software]. http://www.grasshopper3d.com/.

Robert McNeel \& Associates. (2015). Rhinoceros 3D [Software]. http://www.rhino3d.com/.

Sagar, V. R., Wärmefjord, K., \& Söderberg, R. (2018). Geometrical Variation from Selective Laser Heat Treatment of Boron Steels. Procedia CIRP, 75, 409-414. https://doi.org/10.1016/J.PROCIR.2018.04.062

Sigmund, O., \& Maute, K. (2013). Topology optimization approaches. Structural and Multidisciplinary Optimization, 48(6), 1031-1055. https://doi.org/10.1007/s00158013-0978-6

Tam, K.-M. M., \& Mueller, C. T. (2015). Stress Line Generation For Structurally Performative Architectural Design. In L. Combs \& C. Perry (Eds.), Computational Ecologies: Proceedings of the 35th Annual Conference of the Association for Computer Aided Design in Architecture (pp. 94-109). Cincinnati, OH: University of Cincinnati. 